\title{
Trajectory Planning of the live-line maintenance robot manipulator
}

\author{
FENG Ansong ${ }^{1, a}$, WANG Qinghui ${ }^{1, b}$ and GE Xiaoyu ${ }^{1, c}$ \\ ${ }^{1}$ Department of Information Engineering Shenyang University of Chemical Technology \\ Shenyang, Liaoning Province, China \\ a fengansong@sina.com, bwangqh8008@vip.sina.com, cgexiaoyu0301@163.com
}

Keywords: The live-line maintenance robots; trajectory plan; Cubic polynomial interpolation algorithm.

\begin{abstract}
Many countries are conducting research on the live-line maintenance robots. Trajectory plan of the manipulator is introduced. Propose a method of trajectory plan. Using Cubic polynomial interpolation algorithm, the joint angle solved by inverse solution can be determined by plan_ algorithm, and the plan curves are achieved.
\end{abstract}

\section{Introduction}

With the society's development, the demand for the stable power supply is more and more stringent and outage-free maintenance is more and more frequent. Despite many methods to enhance the safety of outage-free maintenance are applied, it is still dangerous to touch the line directly. Because of the importance of safety, many countries are conducting research on the live-line maintenance robots. In 1984, the TOKYO electric company cooperated with TOSHIBA FUJI, FURUKAWA electrical company, FUJIKURA electrical company and AICHI electric company to begin to take the live-line maintenance robot in high sky and high voltage to the work of the research and development[1,2,3,4,5]. In other countries such as CANADA, the United States, FRANCEE and SPAIN, the live-line maintenance robot are also introduced to work in the early 1980s.

In China, the power supply bureau have strong requirement of the live-line maintenance robot. As the price of the imported foreign robot is too high, some power bureau hoped to cooperate with universities and colleges, scientific research units to develop independent intellectual property rights live-line maintenance robot. The ChangSha power bureau and the national university of defense technology proposed city high voltage distribution charged to the feasibility study report homework robot in 1991 .In 1992,Anshan power bureau and Shenyang institute of automation Chinese academy of sciences proposed the project report of the live-line maintenance robot in $10 \mathrm{kv}$ distribution line.

Manipulator is the part of the live-line maintenance robot. The control issues are two problems that the Trajectory planning and movement control. To complete some operation work, the path must be planned in the robot system. The Trajectory planning is stochastic important problem in the robot system. In the paper, the Trajectory planning of the live-line maintenance robot is researched.

\section{Trajectory Planning algorithms of Manipulator}

Robot Trajectory Planning is lower planning and doesn't been involved by Artificial Intelligence problem. The trajectory Planning is in Joint space and Cartesian space, but the trajectory function must be smooth, continuous and operation of the movement of arms. In the paper, the trajectory Plan in Cartesian space is researched.

The motion node of Manipulator is expressed by the position and orientation of the tool coordinate system relate to the job coordinate system. To acquire the trajectory in the joint, first the motion node is transferred to the angle value of joint by inverse resolve of Manipulator; then a smooth function of every joint is fitted curve to make start from starting point to end point and pass through all nodes. The running time of every joint is same in the same motion track. It ensures that all joint arrive the motion node and end point at the same time. 
When the joint is interpolated computed, the constraint condition must be conformed to. The displacement, velocity and acceleration of the joint in the running track must be continuous and the extreme value must be in the tolerance range. The different type interpolation algorithms of Joint are selected to process the different track by conform to the according constraint condition. The interpolation algorithms of Joint trajectory are too much and the cubic polynomial interpolation algorithms are discussed.

In the motion of Manipulator, the start point angle $\theta_{0}$ is known, the endpoint angle $\theta_{f}$ is solved by the kinematics inverse resolve. The track function $\theta(t)$ is expressed by the smooth interpolation function about the start point joint angle and end point joint angel. At $t=0$, the value of $\theta(t)$ is the value $\theta_{0}$ of the start point joint angle. At the time of end point $t_{f}$, the value of $\theta(t)$ is the value $\theta_{f}$ of the end point joint angle. Obviously, many smooth functions are used as joint interpolation function.

To achieve the smooth movement of the single joint, the track function is conformed to 4 constraint conditions at least, the two of which is the joint angle of the start point and the end point, as shown $\theta(0)=\theta_{0}, \theta\left(t_{f}\right)=\theta_{f}$. To satisfy with the continuity of the joint velocity, the others two constraint conditions is the joint velocity of the start point and the end point, as shown $\dot{\theta}(0)=0, \dot{\theta}\left(t_{f}\right)=0$. The four constraint conditions ensure a Cubic polynomial one and only, as shown in formula 1.

$$
\theta(t)=a_{0}+a_{1} t+a_{2} t^{2}+a_{3} t^{3}
$$

The joint velocity and acceleration in the motion track is

$$
\left.\begin{array}{l}
\dot{\theta}(t)=a_{1}+2 a_{2} t+3 a_{3} t^{2} \\
\ddot{\theta}(t)=2 a_{2}+6 a_{3} t
\end{array}\right\}
$$

Make formula 1 and 2 into the corresponding constraints, the four linear equations about $a_{0}, a_{1}, a_{2}$ and $a_{3}$ is acquire, as shown in formula3.

$$
\left.\begin{array}{l}
\theta_{0}=a_{0} \\
\theta_{f}=a_{0}+a_{1} t_{f}+a_{2} t_{f}{ }^{2}+a_{3} t_{f}{ }^{3} \\
0=a_{1} \\
0=a_{1}+2 a_{2} t_{f}+3 a_{3} t_{f}{ }^{2}
\end{array}\right\}
$$

Solve equations set, acquire formula 4 .

$$
\left.\begin{array}{l}
a_{0}=\theta_{0} \\
a_{1}=0 \\
a_{2}=\frac{3}{t_{f}^{2}}\left(\theta_{f}-\theta_{0}\right) \\
a_{3}=-\frac{2}{t_{f}{ }^{3}}\left(\theta_{f}-\theta_{0}\right)
\end{array}\right\}
$$

The joint velocity of the track node is set according to demand., the method of set the Cubic polynomial interpolation algorithms is same as the previous formula and only the velocity constraint conditions transfer as: $\dot{\theta}(0)=\dot{\theta}_{0}, \dot{\theta}\left(t_{f}\right)=\dot{\theta}_{f}$. The four equations that define the Cubic polynomial is showed as formula5. 


$$
\begin{aligned}
& \theta_{0}=a_{0} \\
& \theta_{f}=a_{0}+a_{1} t_{f}+a_{2} t_{f}^{2}+a_{3} t^{3}{ }_{f} \\
& \dot{\theta}_{0}=a_{1} \\
& \dot{\theta}_{f}=a_{1}+2 a_{2} t_{f}+3 a_{3} t_{f}^{2}
\end{aligned}
$$

Solve the equations sets (5) and acquire the coefficient of the Cubic polynomial. as shown in formula 6 .

$$
\left.\begin{array}{l}
a_{0}=\theta_{0} \\
a_{1}=\dot{\theta}_{0} \\
a_{2}=\frac{3}{t^{2}{ }_{f}}\left(\theta_{f}-\theta_{0}\right)-\frac{2}{t_{f}} \dot{\theta}_{0}-\frac{1}{t_{f}} \dot{\theta}_{f} \\
a_{3}=-\frac{2}{t^{3}{ }_{f}}\left(\theta_{f}-\theta_{0}\right)+\frac{1}{t^{2}{ }_{f}}\left(\dot{\theta}_{0}+\dot{\theta}_{f}\right)
\end{array}\right\}
$$

The Cubic polynomial that is defined and describes the motion track that the start point and end point velocity is arbitrary.

\section{Trajectory planning of the live-line maintenance robot manipulator}

The D-H parameter table of the live-line maintenance robot manipulator and the angle value of every joint is as shown table1.The realized step of Trajectory Planning is as shown:

\begin{tabular}{|c|c|c|c|c|c|c|c|}
\hline joint & Coor-dinate & Start value & $\theta_{i}$ & $\alpha_{i}$ & $d_{i}(\mathrm{~mm})$ & $a_{i}(\mathrm{~mm})$ & Motion range \\
\hline 1 & 0 & 90 & $\theta_{1}$ & 90 & 0 & 0 & \pm 180 \\
\hline 2 & 1 & 90 & $\theta_{2}$ & -90 & 215 & 0 & \pm 180 \\
\hline 3 & 2 & 0 & $\theta_{3}$ & 0 & 0 & 550 & $-45 \sim 180$ \\
\hline 4 & 3 & -90 & $\theta_{4}$ & -90 & 0 & 144 & -178 \\
\hline 5 & 4 & 0 & $\theta_{5}$ & 90 & 540 & 0 & \pm 145 \\
\hline 6 & 5 & 0 & $\theta_{6}$ & -90 & 0 & 0 & \pm 180 \\
\hline 7 & 6 & 0 & $\theta_{7}$ & 0 & 200 & 0 & \pm 180 \\
\hline
\end{tabular}

\section{Select interpolation algorithms}

In fact, the velocity of the track node isn't zero, so the Cubic polynomial interpolation algorithms passing through the track node is selected.

\section{Compute the interpolation function}

(1)Select the velocity of track node. Ensure the velocity of track node by inverse resolve according to transient velocity in the tool coordinate system.

(2)Compute interpolation

In figure $1, x, y, z$ express the position of manipulator terminator, the unit is $\mathrm{mm}$. $\mathrm{x}$-coordinate is time unit. In figure2, $x_{i}$ expresses the angle of the manipulator $\theta_{i}, \mathrm{x}$-coordinate is time unit.

\section{Trajectory Real-time generation}

The flow diagram of Trajectory Real-time generation is as shown figure3. $\Delta t$ is refresh frequency of Trajectory, $t_{d}$ is the time of Trajectory end point.

\section{Summary}


In the paper, the Cubic polynomial interpolation algorithms passing through the track node is selected to compute the Trajectory planning of the live-line maintenance robot manipulator in fact. The paper completes the Trajectory planning and simulates it.

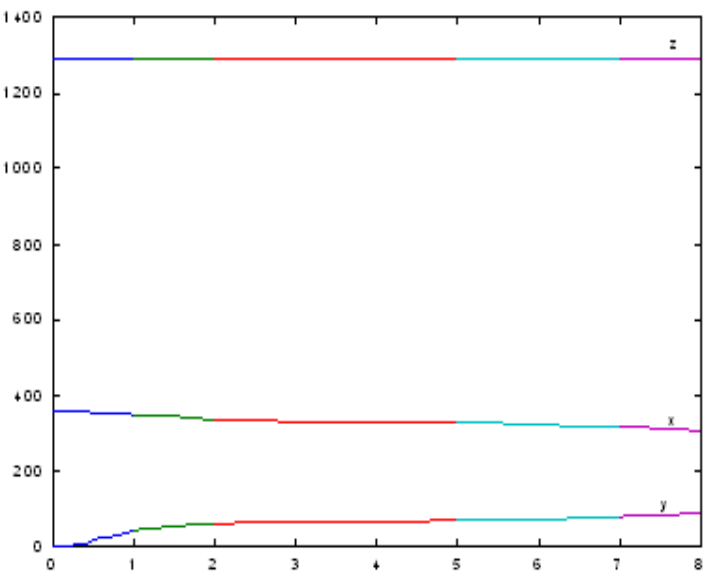

Fig 1 the position of manipulator terminator

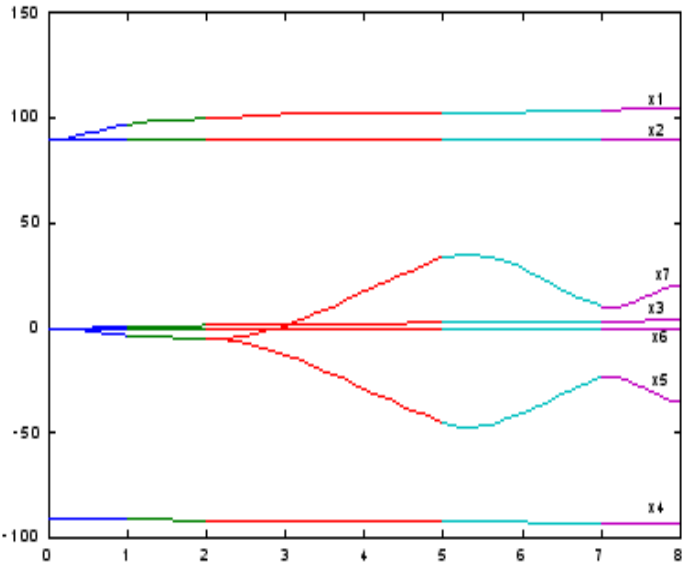

Fig 2 the curve of the joint angel

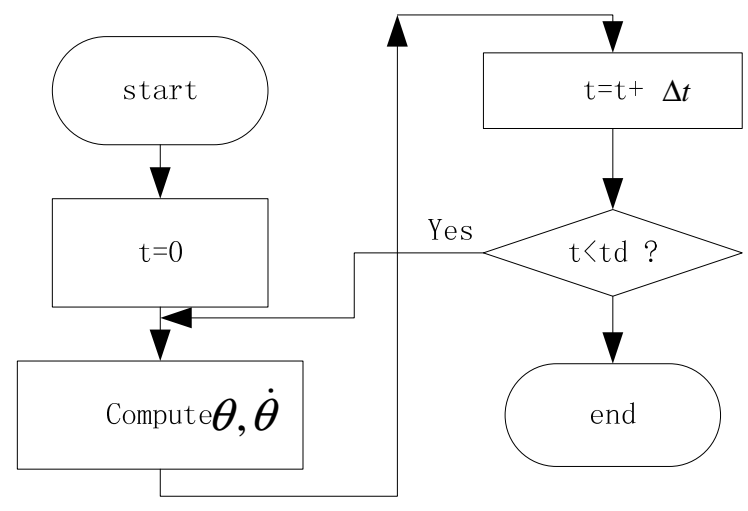

Fig 3 The flow diagram of Trajectory generation

\section{References}

[1] Y. Maruyama, H. Mori : A Hot-Line Manipulator Remotely Operated by the Operator on the ground, ESMO-93,1993;pp,437-444,1993.

[2] Keiichi, Kazuhiko, Hitoshi: Development of the Fully-Automatic live-line Maintenance robot-Phase III, Proceedings of the 4th IEEE International Symposium on assembly and task Planning Soft research Park,Fukuoka,japan,pp, 423-428,May 28-29,2001.

[3] T.toratani,H.yokoyama,S.waki: Manipulator System for Constructing overhead Distribution Lines, IEEE Internal Workshop on Robot and human Communacition,1992,pp235-240.

[4] H.Yokoyama,M.Mukalda,A.Uchlyama: Manipulator System for Constructing Overhead Distribution Lines,pp.445-457,ESMO-93,1993.

[5] D.Faucher: Ground Operated Teleoperation System for Live Power Line Maintenance,Systems Man and Cybernetics 1996 IEEE international Conference On Published ,pp.792-798,1996. 